\title{
An Approximate Point-based Alternative for the Estimation of Variance under Big BAF Sampling
}

Thomas B. Lynch ( $\square$ tom.lynch@okstate.edu )

Oklahoma State University

Jeffrey H Gove

USDA Forest Service Northern Research Station

Timothy G Gregoire

Yale School of Environment, Yale University

Mark J Ducey

Department of Natural Resources and the Environment, University of New Hampshire

Research

Keywords: Bitterlich Sampling, Delta Method, Double Sampling, Forest Inventory, Horizontal Point Sampling, Variance of a Product

Posted Date: December 28th, 2020

DOI: https://doi.org/10.21203/rs.3.rs-135016/v1

License: (1) (1) This work is licensed under a Creative Commons Attribution 4.0 International License. Read Full License 


\section{Abstract}

\section{Background}

A new variance estimator is derived and tested for big BAF (Basal Area Factor) sampling which is a forest inventory system that utilizes two BAF sizes, a small BAF for tree counts and a larger BAF on which tree measurements are made usually including $\backslash \mathrm{dbh} s$ and heights needed for volume estimation.

\section{Methods}

The new estimator is derived using the $\backslash \mathrm{Dm} \backslash$ from an existing formulation of the big BAF estimator as consisting of three sample means. The new formula is compared to existing big BAF estimators including a popular estimator based on Bruce's formula.

Results

Several computer simulation studies were conducted comparing the new variance estimator to all known variance estimators for big BAF currently in the forest inventory literature. In simulations the new estimator performed well and comparably to existing variance formulas.

\section{Conclusions}

A possible advantage of the new estimator is that it does not require the assumption of negligible correlation between basal area counts on the small BAF factor and volume-basal area ratios based on the large BAF factor selection trees, an assumption required by all previous big BAF variance estimation formulas. Although this correlation was negligible on the simulation stands used in this study, it is conceivable that the correlation could be significant in some forest types, such as those in which the Idbh-height relationship can be affected substantially by density perhaps through competition. We also mathematically derived expressions for bias in the big BAF estimator that can be used to show the bias approaches zero in large samples on the order of $1 / n$ where $n$ is the number of sample points.

\section{Full Text}

This preprint is available for download as a PDF.

\section{Figures}




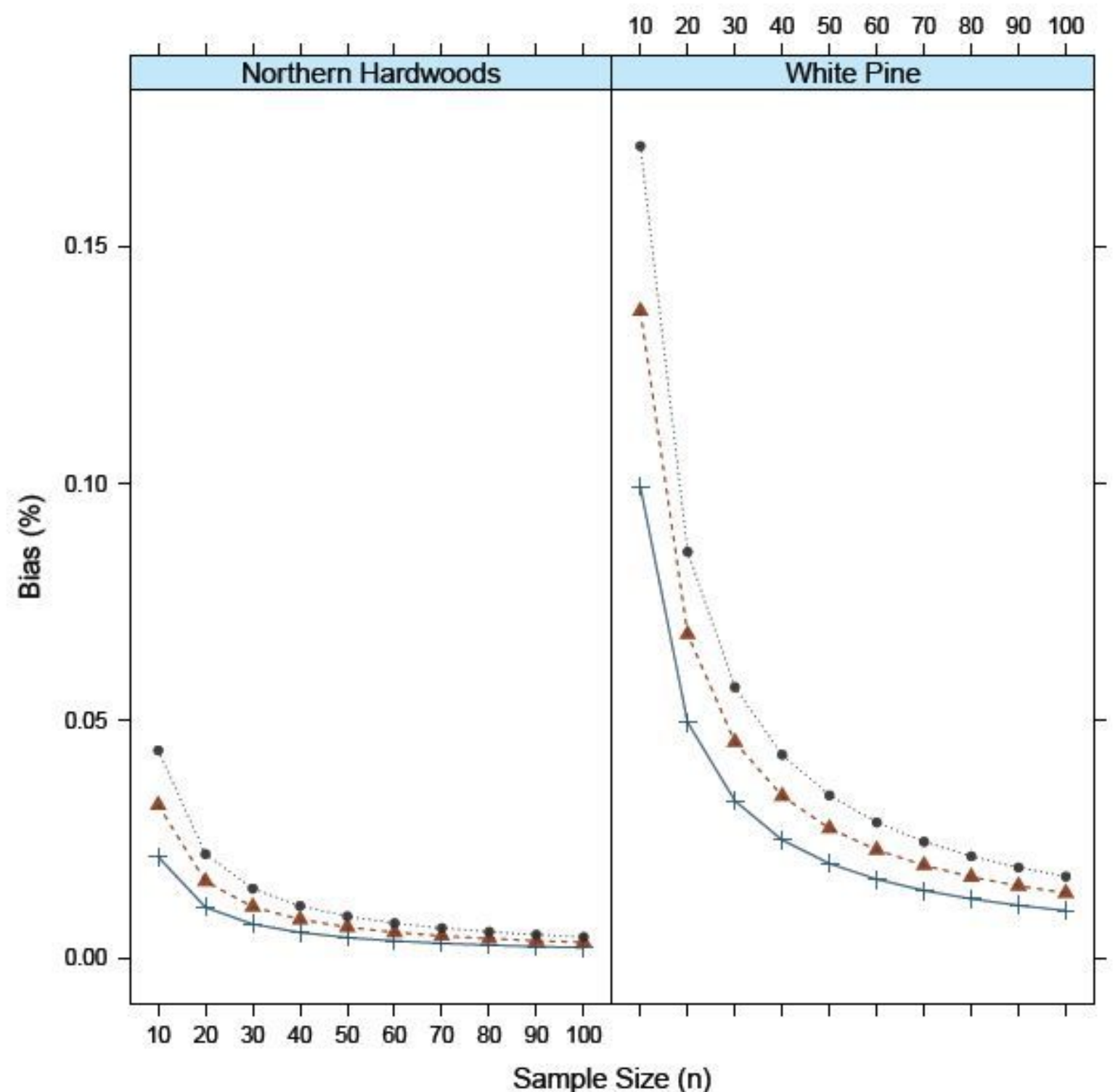

Figure 1

Approximate bias in the big BAF estimator for the northern hardwoods and white pine populations with BAFv $=30$ (dotted, $\bullet$ ), BAFv $=20($ dash, $\Delta$ ) and BAFv $=10$ (solid, + ). 


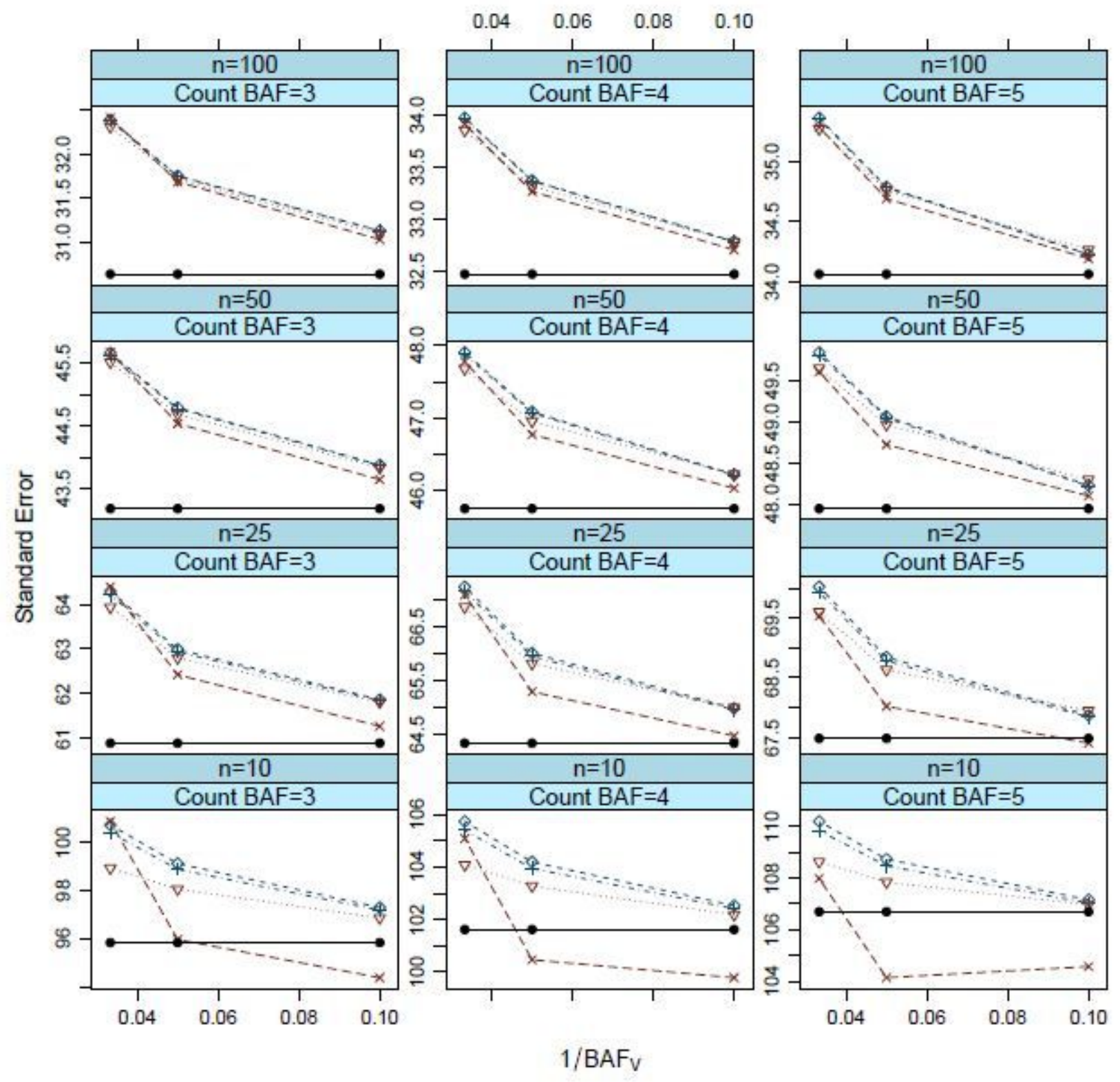

Figure 2

The northern hardwood Monte Carlo standard error simulation results as the average over 1;000 replications for each BAF pair and sample size with the Delta Method (dashed, $\diamond)$, Goodman's (dotdashed, + ), the point-based Delta method (dotted, $\Delta$ ) and the simplied point-based Delta method (longdash, $x$ ). The reference line (solid, $\mathbf{\bullet}$ ) is the average Monte Carlo standard error for the BAFc HPS results. 


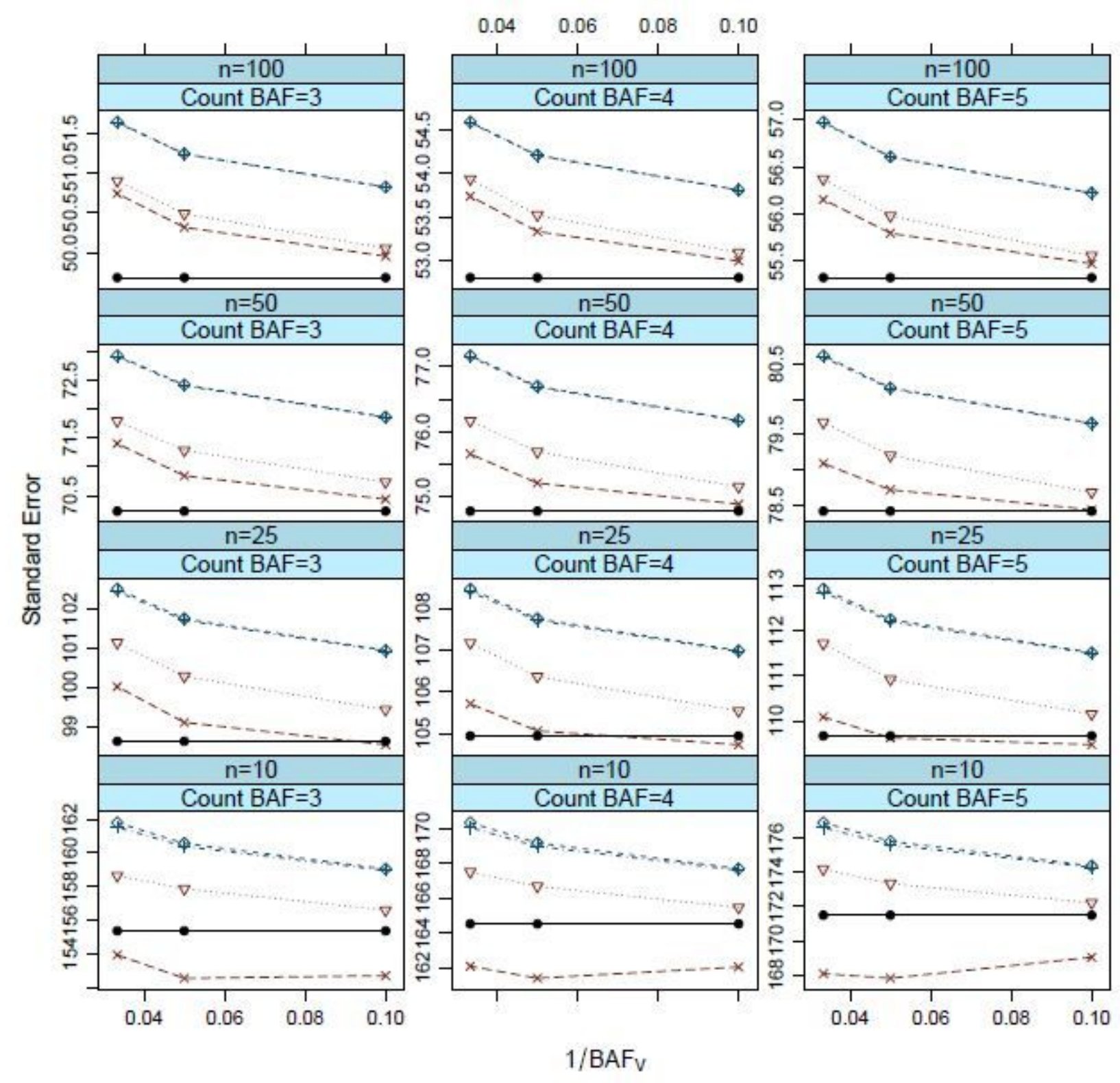

Figure 3

The white pine Monte Carlo standard error simulation results as the average over 1; 000 replications for each BAF pair and sample size with the Delta Method (dashed, $\diamond)$, Goodman's (dot-dashed, + ), the pointbased Delta method (dotted, $\Delta$ ) and the simplied point-based Delta method (long-dash, $x$ ). The reference line (solid, $\mathbf{0}$ ) is the average Monte Carlo standard error for the BAFc HPS results. 


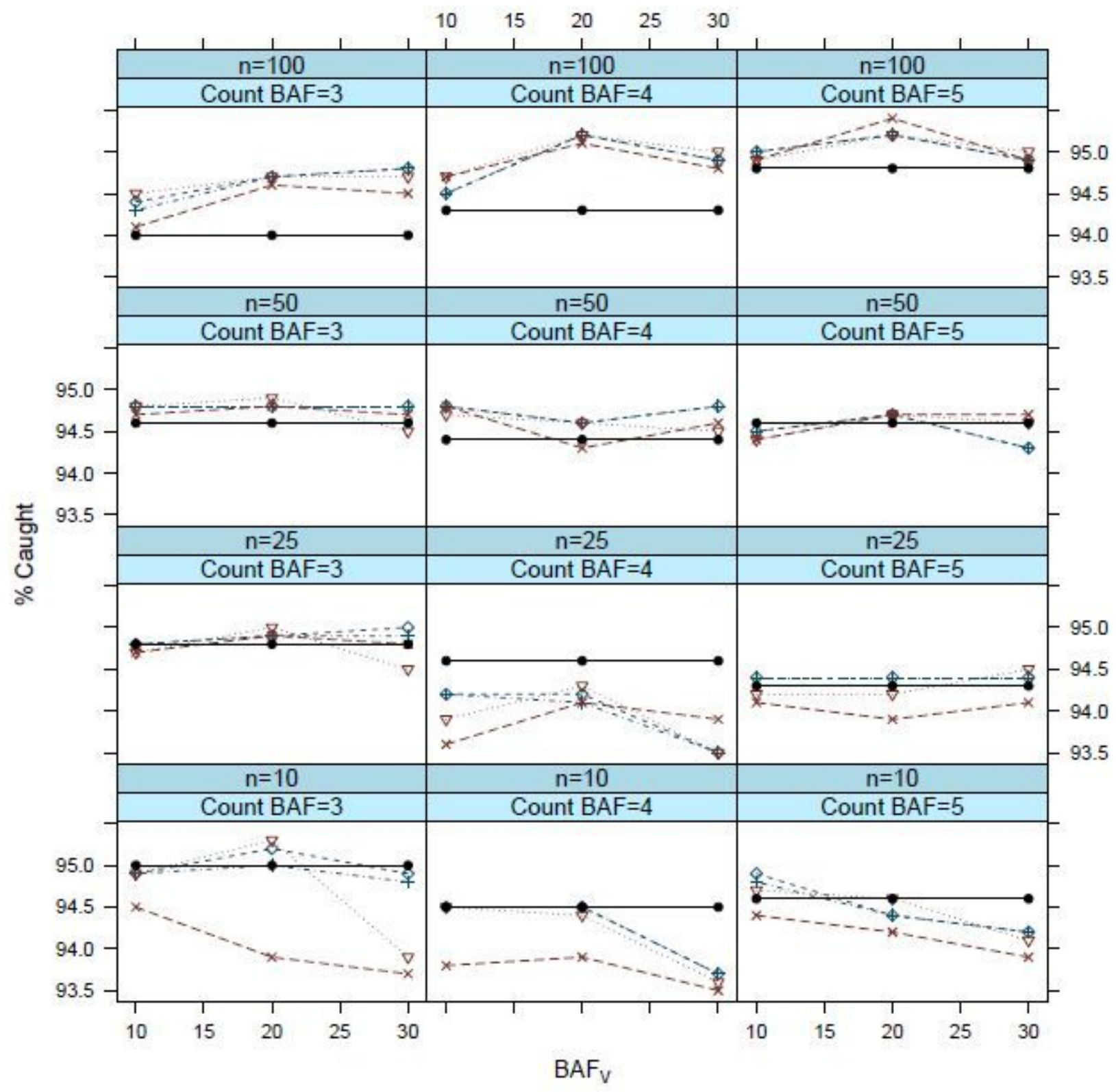

Figure 4

The northern hardwoods Monte Carlo simulation results for condence interval capture rates as the average over 1; 000 replications for each BAF pair and sample size with the Delta Method (dashed, $\diamond)$, Goodman's (dot-dashed, + ), the point-based Delta method (dotted, $\Delta$ ) and the simplied point-based Delta method (long-dash, $x$ ). The reference line (solid, $\bullet$ ) is the average Monte Carlo standard error for the BAFc HPS results. 


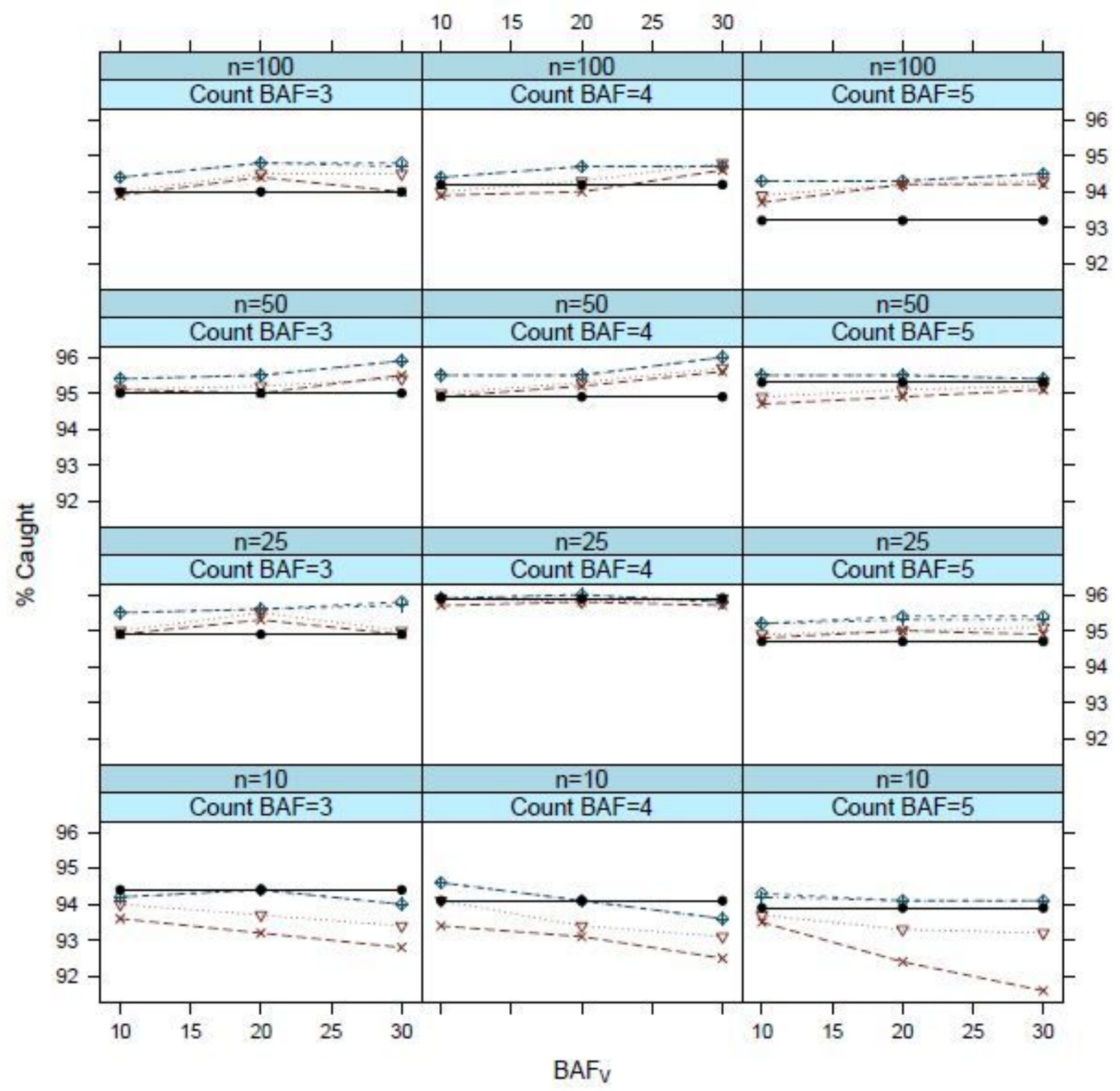

Figure 5

The white pine Monte Carlo simulation results for condence interval capture rates as the average over 1; 000 replications for each BAF pair and sample size with the Delta Method (dashed, $\diamond)$, Goodman's (dotdashed, + ), the point-based Delta method (dotted, $\Delta$ ) and the simplied point-based Delta method (longdash, $x$ ). The reference line (solid, $\mathbf{0}$ ) is the average Monte Carlo standard error for the BAFc HPS results.

\section{Supplementary Files}

This is a list of supplementary files associated with this preprint. Click to download.

- PBDMSupplementaryMaterial.pdf 